\title{
Pneumatic dilation for achalasia: A centimetre outside the comfort zone?
}

\author{
Christopher N Andrews MD MSc FRCPC
}

A chalasia is an uncommon disorder, but most luminal gastroenterologists encounter a few cases each year. Because the etiology is still not fully understood, the management of this disease has not changed much over the years. The goal of achalasia treatment is to disrupt the muscle fibres of the lower esophageal sphincter, without causing a perforation, so that food and liquids may pass through. All of the treatments are palliative in that they relieve symptoms of obstruction but do not address the underlying pathophysiology. Botulinum toxin injection, while simple and well tolerated, lasts only a short time and, thus, is now mainly used only in frail or elderly patients. Experimental endoscopic myotomy has been described in animals (1) but is a long way from clinical adaptation. Surgical esophagomyotomy (ie, Heller's myotomy) and dilation have both been used for a century or longer. With substantial refinements such as laparoscopic access and pneumatic dilation (PD) with visualization, the latter two procedures continue to be the mainstay of definitive therapy for achalasia.

The controversy over the ideal treatment has simmered over the years, often with surgeons advocating surgery and gastroenterologists advocating PD. Even now, head-to-head randomized trials of $\mathrm{PD}$ versus surgery continue. A recent meta-analysis (2) showed that both treatments are effective, with the trade-off of better long-term results ascribed to surgery than with a less invasive procedure but higher perforation rates with PD. In the analysis, 58\% of patients had symptomatic improvement at three years compared with $89 \%$ for laparoscopic Heller's myotomy. In the experience of many practitioners, young people typically choose surgery and older people, who may not require as durable a response, prefer dilation. However, it appears that fewer gastroenterologists are performing PD for reasons that are not entirely clear. Ultimately, the treatment method usually depends on local expertise.

Dilation technology has improved no less than surgical technology over the years. In the past, fixed-diameter dilators and a variety of mercury-weighted bougies, hydrostatic dilators and pneumatic dilators were used with varying degrees of success. Currently, the most safe and effective dilation method for achalasia is to use controlled pneumatic pressure devices, with a $30 \mathrm{~mm}$ Rigiflex balloon (Boston Scientific, USA) being the most commonly used.

Typically, PD is performed over a guidewire under fluoroscopic guidance. This allows for assessment of the progress of the dilation as the narrowed 'waist' of the lower esophageal sphincter disappears. However, in addition to the radiation exposure, this requires bulky and expensive fluoroscopy equipment, which may be in high demand or not available for general use.

Performing PD under direct endoscopic visualization is a good alternative to fluoroscopic guidance. After placement of the dilator over the guidewire, the endoscope is reinserted into the esophagus. The balloon placement and dilation effects are observed proximal to the balloon. This method has been performed for more than 20 years (3), and the American Society of Gastrointestinal Endoscopy has suggested that either visualization method is acceptable for safe dilation in achalasia (4). A second method, whereby the endoscope is placed in the stomach and the dilation is observed from a retroflexed position, has been reported (5). However, this method, in which the endoscope lies across the gastroesophageal junction during the dilation, incurs the disadvantages of a larger dilation diameter (and presumably risk of perforation) as well as unequal radial force from the balloon, which may lead to incomplete muscle fibre rupture. Thus, the latter method is not recommended.

So, if PD with endoscopic guidance is equivalent to fluoroscopic guidance, with the added advantage of no radiation, why are so few gastroenterologists performing dilations for achalasia?

First is the issue of size, also known as the 'fear factor'. Most luminal gastroenterologists are comfortable performing typical balloon dilations of up to $20 \mathrm{~mm}$. Through-the-scope technology is simple and reliable, and it allows for a controlled dilation under direct visualization. However, a perceived excessive risk of complications with a larger dilation (30 mm or greater) may hamper enthusiasm for performing this procedure. PD studies have shown a mean perforation rate of $1.6 \%$ (range $0 \%$ to $8 \%$ ) compared with $0.7 \%$ perforation with clinical manifestations in laparoscopic myotomy trials (mean complication rate of $6.3 \%$ overall) (2). There was also a measurable, albeit small, mortality risk of $0.1 \%$ with surgery.

To put the perforation rate of $1 \%$ to $5 \%$ in perspective, current Canadian guidelines for endoscopic retrograde cholangiopancreatography (ERCP) training aim to keep the complication rate from this procedure to less than $10 \%$ and the mortality to less than $1 \%$ (6). Granted, the patient population undergoing ERCP may be sicker, but it underlines the fact that definitive treatments have a known complication rate that will occur

Division of Gastroenterology, Faculty of Medicine, University of Calgary, Calgary, Alberta

Correspondence: Dr Christopher N Andrews, Centre for Digestive Motility, Division of Gastroenterology, Faculty of Medicine, University of

Calgary, Room 6D24, Teaching, Research and Wellness Building, 3280 Hospital Drive Northwest, Calgary, Alberta T2N 4 N1 .

Telephone 403-592-5020, fax 403-592-5090, e-mail candrews@ucalgary.ca

Received and accepted for publication February 24, 2009 
even in the best of hands. Provided they receive full disclosure of the risks and options, the perforation rate will be acceptable to many patients compared with that of surgery.

A parallel issue is the 'hassle factor'. For a busy clinician, it is often preferable to perform routine endoscopy than to complete a 'white knuckle' procedure that takes more time but is not remunerated accordingly. PD patients also require hours of clinical follow-up that ties up a recovery bed. Even if fluoroscopic guidance is not used, diagnostic imaging services are often needed anyway for a gastrografin esophagram after the procedure.

The final limiting factor is the relative rarity of achalasia. It is generally understood that performing more of a given endoscopic procedure will lead to lower complication rates and that a certain number of supervised procedures are required to reach a competence threshold (7). Because even busy clinical therapeutic endoscopists may perform only one PD per month, the likelihood that gastroenterology trainees will become comfortable performing this procedure is low. Furthermore, trainees may never even see a PD performed during their residency.

In the end, these factors may lead to the demise of PD in many centres across Canada. Although concentrating less common and technically difficult procedures in the hands of a limited number of clinicians is worthwhile (such as ERCP by therapeutic endoscopists), PD is an extension of a procedure that many perform on a routine basis. Proficiency during fellowship training should be encouraged, especially for advanced trainees in therapeutic endoscopy and motility. Otherwise, the paucity of PD practitioners puts the procedure in jeopardy of becoming 'lost'. Surgery then becomes the default procedure and gastroenterologists will consequently have one less procedure in their endoscopic armamentarium.

\section{REFERENCES}

1. Pasricha PJ, Hawari R, Ahmed I, et al. Submucosal endoscopic esophageal myotomy: A novel experimental approach for the treatment of achalasia. Endoscopy 2007;39:761-4.

2. Campos GM, Vittinghoff E, Rabl C, et al. Endoscopic and surgical treatments for achalasia: A systematic review and meta-analysis. Ann Surg 2009;249:45-57.

3. Levine ML, Dorf BS, Moskowitz G, Bank S. Pneumatic dilatation in achalasia under endoscopic guidance: Correlation pre- and postdilatation by radionuclide scintiscan. Am J Gastroenterol 1987;82:311-4.

4. American Society for Gastrointestinal Endoscopy. Esophageal dilation. Gastrointest Endosc 1998;48:702-4.

5. Rai RR, Shende A, Joshi A, Mathur A, Nijhawan S. Rigiflex pneumatic dilation of achalasia without fluoroscopy: A novel office procedure. Gastrointest Endosc 2005;62:427-31.

6. Springer J, Enns R, Romagnuolo J, Ponich T, Barkun AN, Armstrong D. Canadian credentialing guidelines for endoscopic retrograde cholangiopancreatography. Can J Gastroenterol 2008;22:547-51.

7. Armstrong D, Enns R, Ponich T, Romagnuolo J, Springer J, Barkun AN. Canadian credentialing guidelines for endoscopic privileges: An overview. Can J Gastroenterol 2007;21:797-801. 


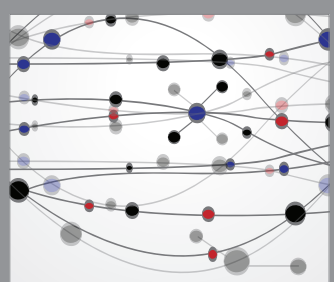

The Scientific World Journal
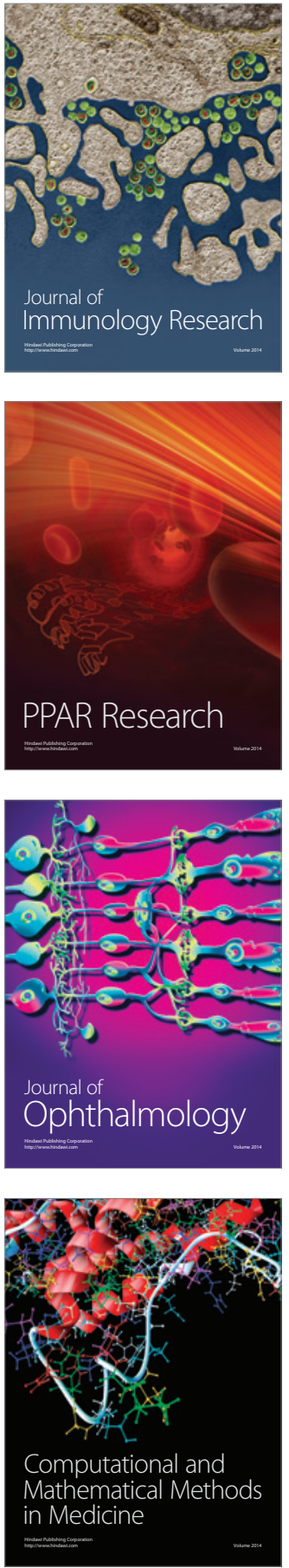

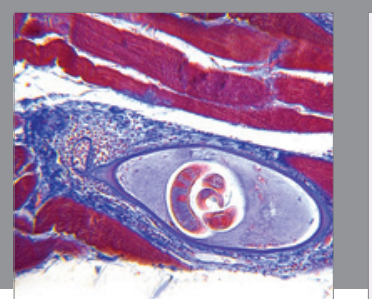

Gastroenterology Research and Practice

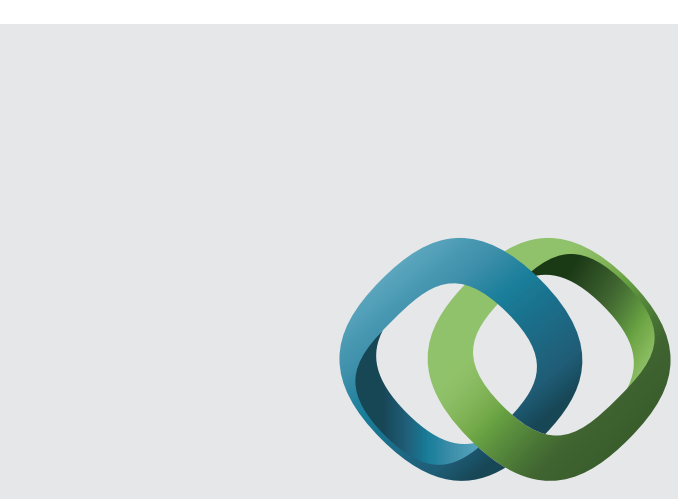

\section{Hindawi}

Submit your manuscripts at

http://www.hindawi.com
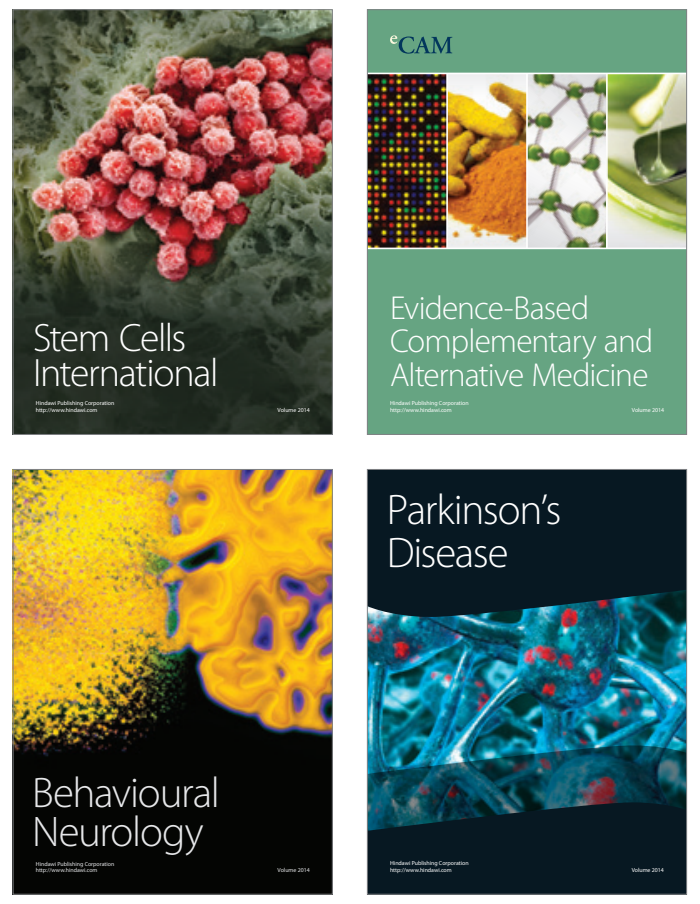
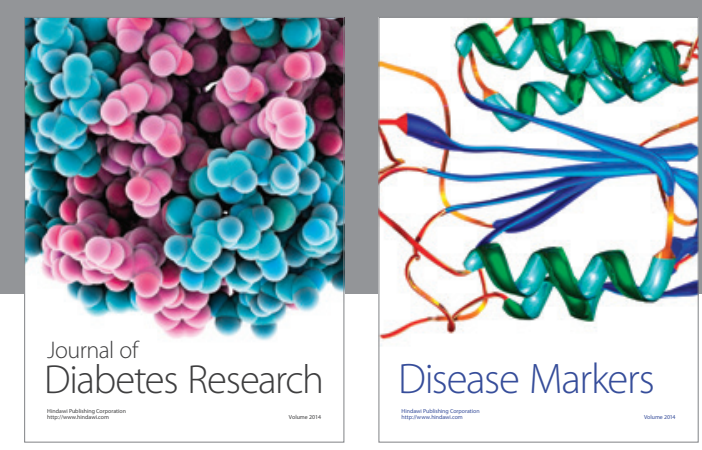

Disease Markers
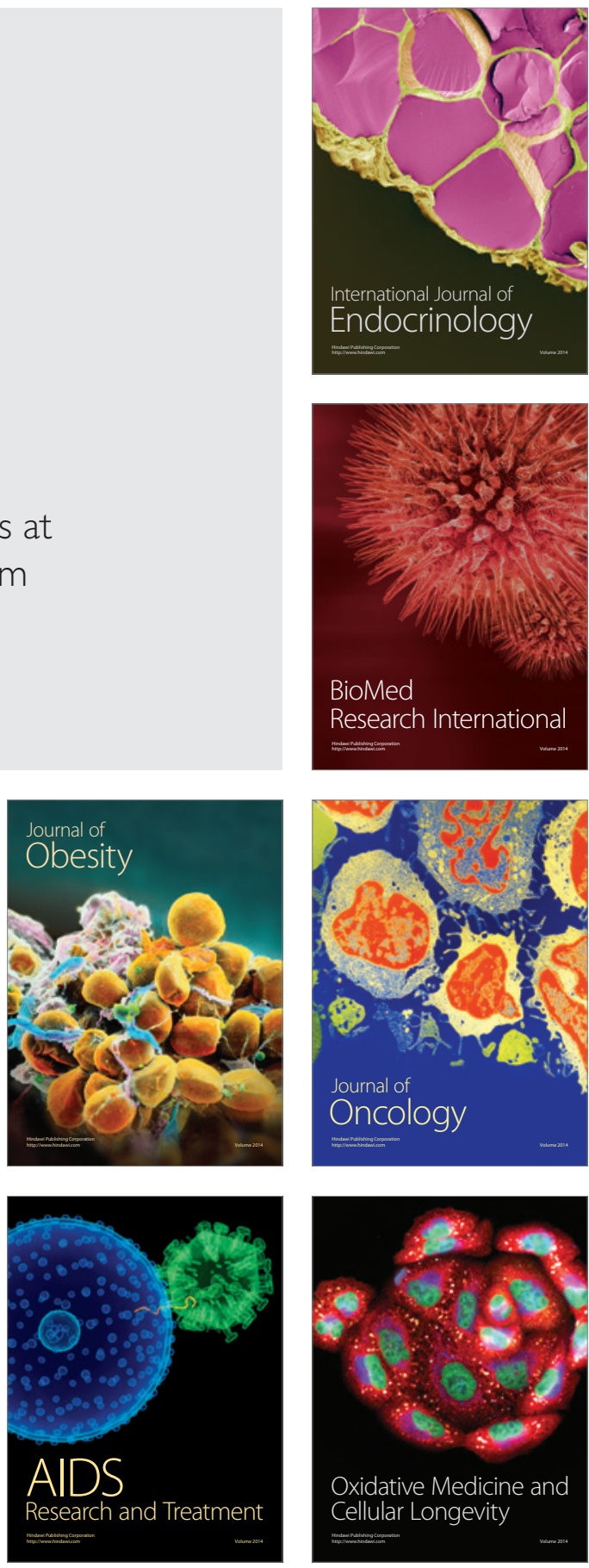